\section{A) Check for updates}

Cite this: J. Anal. At. Spectrom., 2020, 35,1491

DOI: 10.1039/d0ja90028a

rsc.li/jaas

\title{
Correction: Determination of yttrium in titanium alloys using laser-induced breakdown spectroscopy assisted with laser-induced fluorescence
}

\author{
M. Shen, ${ }^{a}$ C. M. Li, ${ }^{b}$ D. Na, ${ }^{c}$ Z. Q. Hao, ${ }^{a}$ X. Y. Li, ${ }^{\star a}{ }^{2}$ L. B. Guo, ${ }^{a}$ Y. F. Lu ${ }^{d}$ and X. Y. Zeng ${ }^{a}$ \\ Correction for 'Determination of yttrium in titanium alloys using laser-induced breakdown spectroscopy \\ assisted with laser-induced fluorescence' by M. Shen et al., J. Anal. At. Spectrom., 2018, 33, 658-662, \\ DOI: 10.1039/C8JA00005K.
}

The authors regret the error in the affiliation of one of the authors, Yongfeng $\mathrm{Lu}$, in the original manuscript. The correct affiliation is: the University of Nebraska-Lincoln (UNL) and not Wuhan National Laboratory for Optoelectronics (WNLO) at the Huazhong University of Science and Technology (HUST). The corrected list of authors and affiliations for this paper is as shown above.

The Royal Society of Chemistry apologises for these errors and any consequent inconvenience to authors and readers.

${ }^{a}$ Wuhan National Laboratory for Optoelectronics (WNLO), Huazhong University of Science and Technology (HUST), Wuhan, Hubei 430074, P. R. China. E-mail: xyli@mail.hust. edu.cn; Fax: +86-27-87541423; Tel: +86-27-87541423

${ }^{b}$ Science and Technology on Surface Physics and Chemistry Laboratory, Mianyang, Sichuan 621700, China

'Institute of Metal Research, Chinese Academy of Sciences, Shenyang, 110016, China

${ }^{d}$ Department of Electrical and Computer Engineering, University of Nebraska, Lincoln, NE, 68588-0511, USA 